\title{
A New Scheduling Algorithm for Reducing Data Aggregation Latency in Wireless Sensor Networks
}

\author{
Meirui Ren ${ }^{1,2}$, Longjiang Guo ${ }^{1,2 \#}$, Jinbao $\mathrm{Li}^{1,2}$ \\ ${ }^{1}$ School of Computer Science and Technology, Heilongjiang University, Harbin, China \\ ${ }^{2}$ Key Laboratory of Database and Parallel Computing of Heilongjiang Province, Harbin, China \\ E-mail: ljguo_1234@sina.com \\ Received April 20, 2010; revised June 21, 2010; accepted July 29, 2010
}

\begin{abstract}
Existing works on data aggregation in wireless sensor networks (WSNs) usually use a single channel which results in a long latency due to high interference, especially in high-density networks. Therefore, data aggregation is a fundamental yet time-consuming task in WSNs. We present an improved algorithm to reduce data aggregation latency. Our algorithm has a latency bound of $16 R+\Delta-11$, where $\Delta$ is the maximum degree and $R$ is the network radius. We prove that our algorithm has smaller latency than the algorithm in [1]. The simulation results show that our algorithm has much better performance in practice than previous works.
\end{abstract}

Keywords: Reducing Latency, Wireless Sensor Networks, Scheduling Algorithm

\section{Introduction}

A wireless sensor network (WSN) consists of sensor nodes that are capable of sensing, processing, and transmitting. Each node is responsible for covering a certain geographical area with the primary functions of monitoring changes and reporting them using its radio transmitter to the sink(base station) and in cooperation with other nodes. Sensory data is sent to the sink in a multi-hop mode.

WSNs have no infrastructure and nodes are self-organized arbitrarily. WSNs have proven their success in a various applications such as battlefield surveillance, traffic monitoring and forest fire monitoring. In some applications, e.g. forest fire monitoring and aquiculture surveillance, end users want to extract data aggregation information from WSNs with low latency.

However, WSNs usually use a single channel, which results in a long latency due to high interference, especially in high-density networks. When two or more sensors send data to a common neighbor at the same time, data

*National Natural Science Foundation of China (NSFC) for Young Scholar under grant No. 60803015; China Postdoctoral Science Foundation under grant No. 20080430902; Science and Technology Innovation Research Project of Harbin for Young Scholar under grant No. 2008RFQXG107; Heilongjiang Postdoctoral Science Foundation under grant No. LRB08-021; Science and Technology Key Research of Heilongjiang Educational Committee under grant No.1154Z1001; Heilongjiang University Fundation for Distinguished Young Scholars; The National Natural Science Foundation of China (No.61070193); the Key Scientific and Technological Research Project of Heilongjiang Province of China (No.GC09A109). collision occurs at the common neighbor, preventing it from successfully receiving any data. The data sent by a sender should be received by a corresponding receiver with no collisions. The receiver aggregates the received data with its own data, and stores the aggregated data as its new data. The time consumed by a single sending-receiving-aggregating-storing is normalized to one, and parallel sending-receivings are desirable for reducing network delay.

In this paper, we focus on reducing the latency of data aggregation by constructing a good schedule with low latency. Minimum Data Aggregation Latency (MDAL) is an important research problem. MDAL is defined as follows: Given a wireless senor network that consists of a number of sensors and a sink, supposing each sensor has a piece of data to be aggregated and transmitted to the sink, the MDAL problem is to design a transmission schedule of data aggregation for all sensors such that there is no conflict between any two concurrent transmissions and the total number of timeslots for all data to reach the sink is minimized.

Extensive research has been conducted on the MDAL problem, such as [1,2]. Chen et al. [2] prove that the MDAL problem is NP-hard. They designed a $(\Delta-1)$-approximation algorithm named SDA (Shortest Data Aggregation) based on Shortest Path Tree for data aggregation with a latency bound of $(\Delta-1) \cdot R$, where $\Delta$ is the maximum degree and $R$ is the network radius. Huang et al. [1] proposed an algorithm based on Maximal Independent Set 
which has a latency bound of $23 R+\Delta-18$. Here $\Delta$ contributes to an additive factor instead of a multiplicative one. The algorithm is a nearly constant approximation and it has a significantly less latency bound than earlier algorithms especially when $\Delta$ is large. In this paper, we present an algorithm relying on reducing the number of blue nodes and finding maximal non-conflicting transmission schedule sets which has a latency bound of $16 R+\Delta-11$. Our algorithm is also a nearly constant approximation and it has a significantly less latency bound than the existing best known algorithm in [1] when $R$ is large.

The remainder of this paper is organized as follows. The formalized MDAL problem and all related work are presented in Section 2. In Section 3, we propose our algorithm and give details of analysis on performance. In Section 4, we evaluate the average performance of the proposed algorithm through simulations and compare it with the algorithm in [1]. Section 5 concludes this paper.

\section{Preliminaries}

In this section, we first present some assumptions and formalize the MDAL problem, and then we discuss all related work in details.

\subsection{Problem Description}

We consider a wireless sensor network consisting of stationary nodes along with one sink node distributed over an Euclidean plane. All the sensor nodes are homogeneous. Each sensor node is equipped with a RF transceiver that can be used to send or receive data. We assume that each sensor node has omni-directional antenna and the transmission coverage of any sensor node is a circle with unit radius centered at the sensor.

We assume that each sensor knows its geometric position in the network. The sink has global knowledge of all the sensors' IDs and positions. Transmission is deterministic and proceeds in synchronous time rounds controlled by a global clock.

In each time round, any node cannot send and receive data simultaneously, i.e. any node either sends data or receives data in a round.

Definition 1 [Neighbor Set] For a sensor node $u$, if there exists another sensor node $v$ such that $v$ lies in $u$ 's transmission area, then $v$ is called $u$ 's neighbor. All of $u$ 's neighbors form a set, which is called $u$ 's Neighbor Set, denoted by Neighbor $(u)$.

Data sent by any sender simultaneously reaches all the nodes in its neighbor set.

Definition 2 [Transmission Schedule] $u \rightarrow v$ is call- ed a Transmission Schedule, where $u$ is called sender, $v$ is called a receiver. $u \rightarrow v$ denotes that $u$ transmits data to $v$.

If two or more nodes are sending in the same round and there exists a node $u$ in their overlapped transmission area, then $u$ cannot successfully receive any data since all transmissions are interfering with each other. This situation is called a collision. For example, in Figure 1(a), there are two ongoing transmission schedules $u \rightarrow v$ and $x \rightarrow v$ in the same round. $v$ will not receive anything. In Figure 1(b), there are two ongoing transmission schedules $u \rightarrow v$ and $x \rightarrow y$ in the same round. $v$ is in $x$ 's transmission range, therefore $v$ will not receive anything.

Definition 3 [Conflicting Transmission Schedules] $u$ $\rightarrow v$ and $x \rightarrow y$ are called Conflicting Transmission Schedules if and only if $v \in \operatorname{Neighbor}(x)$ or $y \in$ Neigh$\operatorname{bor}(u)$.

The main task of a sensor is to collect data and forward aggregated data to the sink, and data can be "aggregated" all the way to the sink. In other words, if a node has received one packet from its neighbor before its scheduled transmission time round, then it can merge this packet with its own data packet and simply waits to send this merged packet later. The situation where packets cannot be merged does exist, and it is called data collection. In this paper we only focus on data aggregation meaning that data can be merged all the way to the sink.

For simplicity, a wireless sensor network with sink node $s$ can be represented as a graph $G=(V, E)$, where $V$ denotes all the sensor nodes in the network and $s \in V$, An edge $(u, v) \in E$ indicates that $u$ lies in $v$ 's transmission range and $v$ lies in $u$ 's transmission range.

A data aggregation schedule is a sequence of transmission schedule sets $\left\{S_{1}, S_{2}, \ldots, S_{l}\right\}$, where $S_{i}(i=1,2, \ldots l)$ is a transmission schedule set satisfying the following conditions:

1) Any two transmission schedules $u \rightarrow v, x \rightarrow y$ in $S_{i}$ $(i=1,2, \ldots l)$ are non-conflicting transmission schedules, i.e. $v \notin \operatorname{Neighbor}(x)$ and $y \notin \operatorname{Neighbor}(u)$.

2) $\forall i \neq j$, Sender $\left(S_{i}\right) \cap \operatorname{Sender}\left(S_{j}\right)=\varnothing$, where Sender $\left(S_{i}\right)$ denotes the sender set of transmission schedules in $S_{i}$.

3) $\bigcup_{i=1}^{l} \operatorname{Sender}\left(S_{i}\right)=V-\{s\}$.

$l$ is called the data aggregation latency.

The MDAL problem is defined as follows. Given a graph $G=(V, E)$ and the sink node $s \in V$, find a data aggregation schedule with the minimum latency.

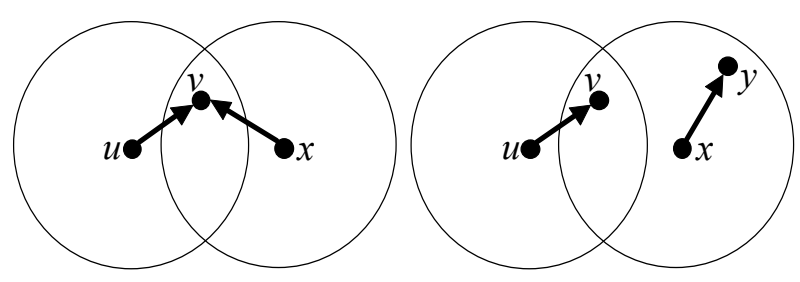

(a)

(b)

Figure 1. Two types of collisions. 


\subsection{Related Work}

Extensive research has been conducted on data aggregation. One category of existing works focuses on how to design an energy efficient data aggregation algorithm. These works of data aggregation focus on energy efficiency. Data aggregation is also called convergecast. Convergecast is about a sensor network with a sink such that all sensor nodes collect data and report to the sink through multi-hop communications. Annamalai et al. [3] designed a heuristic algorithm for both broadcast and convergecast. The convergecast tree constructed in their algorithm can be used for broadcast as well. Upadhyayula et al. [4] designed another heuristic algorithm for convergecast, aiming at reducing energy consumption and latency. These two works mentioned above both proposed heuristic approaches and used simulations to verify their results without giving theoretical analysis. In our work, we verified our results through both simulations and theoretical analysis.

Another category focuses on how to design a conflictfree scheduling. A distributed cross-layer scheduling protocol for data aggregation was proposed in [5], in which each node negotiates with its parent to decide its timeslots for transmission and constructs a schedule for its query processing. Chipara et al. [6] developed a dynamic scheduling scheme supporting different kinds of aggregation queries, assuming that an aggregation tree has already been constructed. Yu et al. [7] studied the energylatency tradeoff of scheduling for data aggregation. Practical issues of data aggregation, especially about the MAC layer, have also been studied in the literature. Huang and Zhang [8] studied packet loss and focused on reliability issues in data aggregation. Zhang et al. [9] addressed the issue of bursty convergecast in real-time applications. The high-volume burst traffic often arises in event-driven applications. These applications require for reliable and real-time packet delivery to the sink. The large number of packets generated within a short period leads to high degree of channel contention and thus a high probability of packet collision. Zhang et al. focused on improving channel utilization and reducing retransmission incurred by channel contention. Krishnamachari et al. [10] studied data aggregation from another aspect. They considered the case where there is a subset of nodes whose data need to be sent to the sink and regard aggregating these data as a way to save energy. Intanagonwiwat et al., in a short paper [11], evaluated the impact of greedy aggregation to increase the amount of path sharing and reduce energy consumption. All the above works aimed at minimizing the overall energy consumption of sensors subject to the latency constraint.

The most relevant works of the MDAL problem are on theoretical side. Kesselman and Kowalski [12] designed a randomized, distributed algorithm with latency $\mathrm{O}(\log$ $n$ ). In their model, it is assumed each node can vary its transmission range to reduce links. Chen et al. [2] designed a $(\Delta-1)$-approximation algorithm called SDA for data aggregation, which has a latency bound of $(\Delta-1) \cdot R$, where $\Delta$ is the maximum degree of the network and $R$ is the network radius. They also proved that the minimum data aggregation time problem is NP-hard. Huang et al. [1] designed an algorithm based on maximal independent sets which has a latency bound of $23 R+\Delta-18$. They reduced the data aggregation latency from a multiplicative factor of $\Delta$ to additive factor. Their algorithm is nearly constant and it has a significantly less latency bound than the previous algorithms when $\Delta$ is large.

In this paper, we present an algorithm based on reducing the number of blue nodes and maximal non-conflicting transmission schedule sets which has a latency bound of $16 R+\Delta-11$. Our algorithm is also nearly constant and it has a significantly less latency bound than the previous algorithms.

\section{Our Data Aggregation Algorithm}

In this section, we present our approximation algorithm. Our algorithm has a data aggregation latency of $16 R+\Delta$ -11 , where $R$ is the network radius and $\Delta$ is the maximum degree of the network. This result is better than the currently best known algorithm [1] whose latency is $23 R$ $+\Delta-18$, since if $\Delta$ and $R$ are both large, our algorithm achieves a smaller latency. Large $\Delta$ and $R$ are frequent especially in large-scale, dense networks. The key behind-the-scene ideas of our algorithm include reducing the number of blue nodes and maximal non-conflicting transmission schedule sets in data aggregation scheduling. Our algorithm has three phases: 1) Construct MIS Layer by Layer; 2) Data Aggregation Tree Construction reducing the number of blue nodes; 3) Data Aggregation Scheduling based on maximal non-conflicting transmission schedule sets.

The details of our algorithm are showed in the next four subsections. To better understand our algorithm, some examples are given in these four subsections.

\subsection{Construct MIS Layer by Layer}

This phase is the same as [1]. For a given graph $G=(V$, $E$ ) and the sink node $s \in V$, a Breadth First Search Tree (BFST) rooted at $s$ for the graph $G$ is firstly constructed. In this phase, BFS starts at sink node $s$, which is the root of BFST at layer 0 , then BFS explores all the neighboring nodes which are added into BFST to be the children of $s$ at layer 1 . Then, the new nodes adjacent to layer 1 nodes are added into BFST at layer 2 to be the children of the nodes at layer 1, and so on. The BFS traversal terminates when every node in $V$ has been visited. For example, Figure 2 shows a topology of $G$. Node 0 is the 
sink. Each circle denotes a sensor node. Node ID lies in each circle.

Figure 3 shows the BFST of $G$ in Figure 2. The number above each node denotes its layer in the BFST. $\mathrm{L}_{1}=$ $\{1,2,3,4,5\}$ denotes that nodes $1,2,3,4,5$ are at layer 1. Similarly, nodes $6,7,8,9,10$ are at layer 2 and nodes $11,12,13,14,15,16,17$ are at layer 3 .

In Figure 3 dashed lines denote the edges in $G$, but they are not in BFST of $G$.

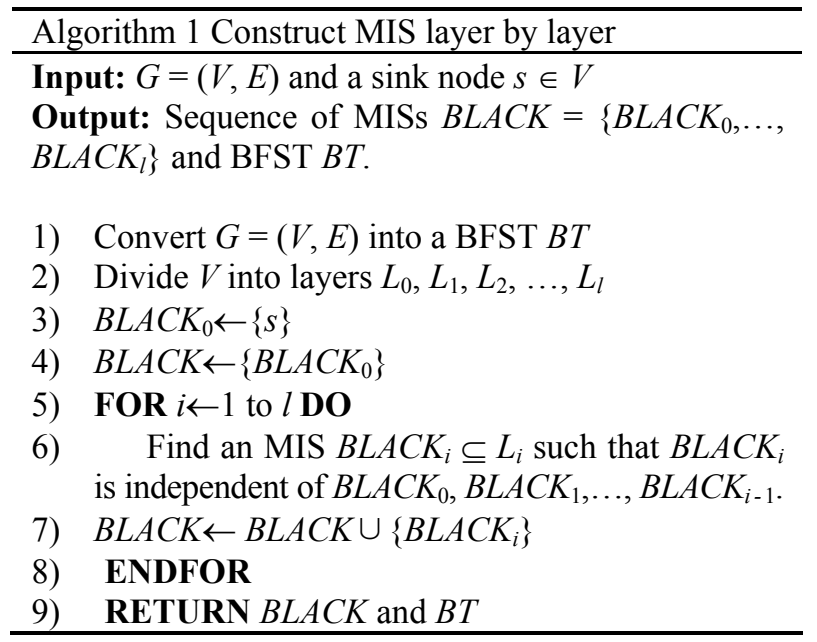

Based on BFST, all nodes are divided into layers $L_{0}$, $L_{1}, L_{2}, \ldots, L_{l}$. We then form a Maximal Independent Set (MIS) layer by layer. This procedure begins from layer 0 . On layer 0 , there is only one node, the sink node $s, s$ for$\mathrm{ms}$ an MIS $B L A C K_{0}=\{s\}$, which is marked as black. We then move on to layer 1 and construct an MIS $B L A C K_{1}$ and mark these nodes black again. Note that $B L A C K_{i}$ must be independent of the MISs from layer 0 to layer $i-1$. This process is repeated until all the layers have been processed. The nodes which are not marked black are marked white at last. The pseudocode of layered MIS construction is given in Algorithm 1 [1].

Figure 4 shows an example which constructs an MIS based on BFST in Figure 3.

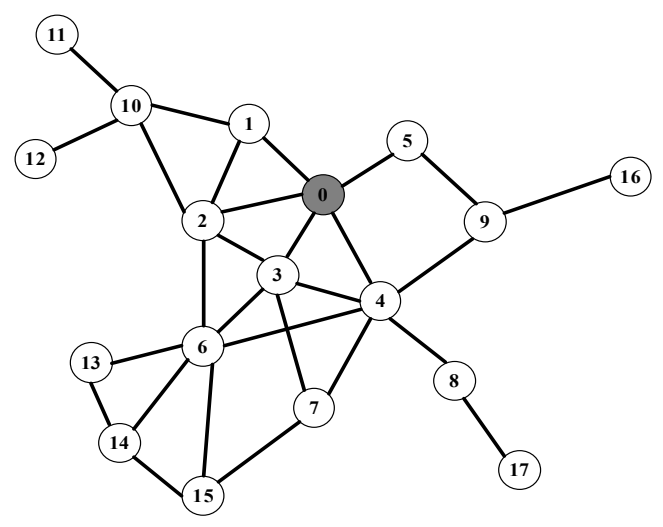

Figure 2. Topology of $G$.

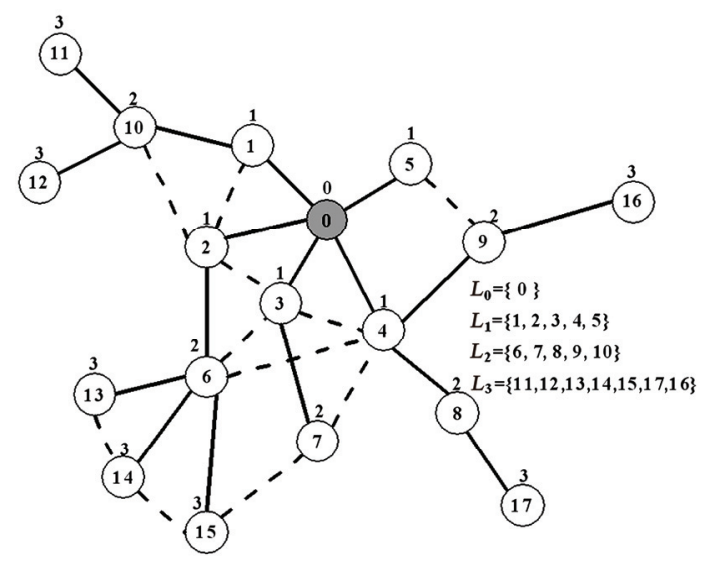

Figure 3. BFST of $G$.

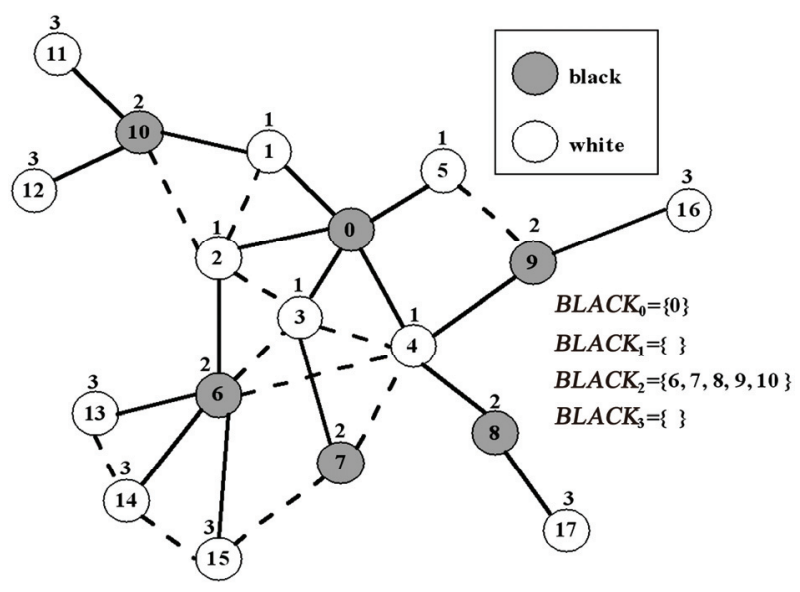

Figure 4. Construct MIS layer by layer.

\subsection{Data Aggregation Tree Construction}

The data aggregation tree construction has two phases: 1) Find a sequence of blue node sets $\left\{B L U E_{1}, B L U E_{2}, \ldots\right.$, $\left.B L U E_{l-1}\right\}$ that connect black nodes layer by layer, where the blue nodes in $B L U E_{i}$ interconnect black nodes in $B L$ $A C K_{i-1}$ and $B L A C K_{i+1}$, at the same time, we also construct a data aggregation tree $D T ; 2)$ Reduce the number of blue nodes layer by layer, meanwhile, we obtain an optimized data aggregation tree. The first phase is similar with the algorithm in [1]. The second phase is an optimized procedure which can reduce data aggregation latency. Through the second phase some blue nodes are converted into white leaves. The latency of data aggregation is reduced from $23 R+\Delta-18$ to $16 R+\Delta-11$. The details of analysis will be presented in Subsection 3.4.

In the first phase, we find a sequence of blue node sets $\left\{B L U E_{1}, B L U E_{2}, \ldots, B L U E_{l-1}\right\}$ that interconnect black nodes layer by layer. To find blue node set $B L U E_{1}$, we look at $B L A C K_{2}$. Each black node in $B L A C K_{2}$ has a parent in BFST $B T$ and this parent must be white since black nodes are independent of each other. These white nodes 
are colored blue and an edge between black node and its white parent is added into data aggregation tree DT. Moreover, the blue node must be connected with the black node $s$ in $B L A C K_{0}$. The edge between the blue node and the black node $s$ is added into $D T$. Note that to find blue node set $B L U E_{i}$, we color the parent of each black node in $B L A C K_{i+1}$ blue. The blue node must be connected with the black node in $B L A C K_{i}$ or $B L A C K_{i-1}$. This process is repeated layer by layer and finally a sequence of blue node sets $B L U E=\left\{B L U E_{1}, B L U E_{2}, \ldots, B L U E_{l-1}\right\}$ and a desired data aggregation tree $D T$ are both obtained. The pseudo code is presented in Algorithm 2.

Figure 5 shows an example of finding a sequence of blue node sets based on the MIS in Figure 4.

In the second phase, we reduce the number of blue nodes layer by layer and get an optimized data aggregation tree. The purpose of reducing the number of blue nodes is to reduce data aggregation latency. Recall the algorithm analysis in [1], the data aggregation latency greatly depends on how many blue nodes lie inside the transmission range of a black node. A small number of blue nodes results in a short data aggregation latency.

Algorithm 2 Finding blue node sets

Input: $G=(V, E)$; Sink node $s \in V$; Sequence of MISs $B L A C K=\left\{B L A C K_{0}, \ldots, B L A C K_{l}\right\}$; BFST $B T$.

Output: Sequence of blue node sets $B L U E=\left\{B L U E_{1}\right.$, $\left.B L U E_{2}, \ldots, B L U E_{l-1}\right\}$ and data aggregation tree $D T$

1) Procedure FindBlueNodeSets $(G, s, B L A C K, B T)$

2) $D T=\left(V_{D T}, E_{D T}\right) ; V_{D T} \leftarrow V ; \quad E_{D T} \leftarrow \varnothing ; B L U E \leftarrow \varnothing$;

3) //Find blue node sets that connect black nodes

4) FOR $i \leftarrow 1$ to $l-1$ DO

5) $\quad$ FOR each black nodes $u \in B L A C K_{i+1} \quad$ DO

6) Find $u$ 's parent $p_{B T}(u)$ in BFST $B T$

7) Color $p_{B T}(u)$ blue

8) $\quad \operatorname{Add} p_{B T}(u)$ to $B L U E_{i}$

9) Add an edge $\left(u, p_{B T}(u)\right)$ to $E_{D T}$

10) Find a black node $v$ which can communicate with $p_{B T}(u)$ from $B L A C K_{i} \cup B L A C K_{i-1}$

11) Add an edge $\left(p_{B T}(u), v\right)$ to $E_{D T}$

12) ENDFOR

13) $B L U E \leftarrow B L U E \cup B L U E_{i}$

14) ENDFOR

15) //Color remaining nodes white/

16) FOR each remaining node $u$ DO

17) Color $u$ white

18) Find $u$ 's parent $p_{B T}(u)$ in BFST $B T$

19) Add an edge $\left(u, p_{B T}(u)\right)$ to $E_{D T}$

20) ENDFOR

21) RETURN $B L U E$ and $D T$

For a black node $u$, we only keep those blue nodes that can communicate with $u$ 's 2-hop black neighbors. For a clear description, some concepts are given as follows.
Definition 4 [Coverage and Coverage Density] For a blue node $v \in B L U E_{i+1}$, a subset of $B L A C K_{i+2}$ Coverage $(v) \subseteq B L A C K_{i+2}$, is called $v$ 's Coverage if $v$ can communicate with each black node in Coverage $(v)$, i.e. $v$ can Cover all black nodes in Coverage(v). The cardinality of Coverage ( $v)$ is called v's Coverage Density.

For a black node $u \in B L A C K_{i}$, we only keep the blue nodes in $B L U E_{i+1}$ that can cover $u$ 's 2-hop black neighbors in $B L A C K_{i+2}$. The following lemma indicates that we can keep at most 13 blue nodes which can cover $u$ 's 2-hop black neighbors in $B L A C K_{i+2}$.

Lemma 3.1 For a black node $u \in B L A C K_{i}(i=0,1, \ldots$, $l-2)$, there are at most 13 blue nodes which can cover $u$ 's 2-hop black neighbors in $B L A C K_{i+2}$.

Proof: For a black node $u \in B L A C K_{i}(i=0,1, \ldots, l-$ $2)$, suppose there are at least 14 blue nodes $c_{1}, c_{2}, \ldots, c_{14}$ which can cover $u$ 's 2 -hop black neighbors in $B L A C K_{i+2}$.

Assume that the transmission radius of a sensor node is 1 . Consider $D_{2 u}$, a circular of radius 2 centered at the black node $u$. All $u$ 's 2-hop black neighbors lie inside $D_{2 u}$. Since black nodes are mutually independent, for each black node in $D_{2 u}$, we consider a circular of radius 0.5 centered at this black node, then all of those circulars must be disjoint. $u$ 's blue children nodes lie inside $D_{1 u}$, a circular of radius 1 centered at the black node $u$.

Since we suppose there are at least 14 blue nodes $c_{1}$, $c_{2}, \ldots, c_{14}$ which can cover $u$ 's 2-hop black neighbors, then each of $u$ 's 2-hop black neighbors can be covered at least by one blue node of those 14 blue nodes. From $u$ 's 2-hop black neighbors, we certainly find at least 14 black nodes $b_{1}, b_{2}, \ldots, b_{14}$ such that $b_{i}(i=1,2, \ldots, 14)$ is covered only by $c_{i}(i=1,2, \ldots, 14)$ and $u$ 's other 2 -hop black neighbors besides these 14 black nodes $b_{1}, b_{2}, \ldots, b_{14}$ are covered at least by two blue nodes. (If we can not find at least 14 black nodes $b_{1}, b_{2}, \ldots, b_{14}$, then there are at most 13 blue nodes which can cover $u$ 's 2-hop black neighbors. The lemma is proved.) These 14 black nodes $b_{1}, b_{2}, \ldots$, $b_{14}$ certainly lie outside the circular $D_{1 u}$ and inside the

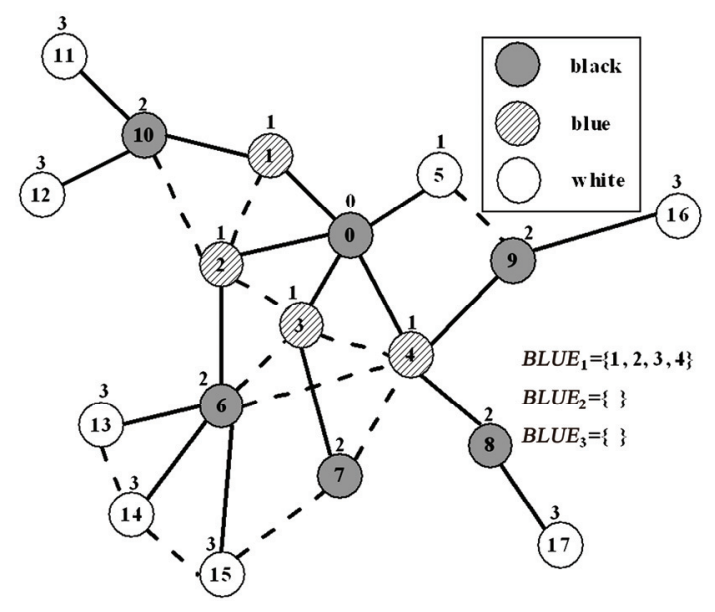

Figure 5. Finding blue node sets. 
circular $D_{2 u}$ centered at the black node $u$. (Refer to Figure 6) We equably divide $D_{2 u}$ into 13 sectors. According to the pigeonhole principle [13], there are two black nodes at least lie inside the same sector, i.e. there are at least 2 circulars of radius 0.5 centered at black nodes lie inside the same sector. Without loss of generality, suppose black nodes $b_{1}$ and $b_{2}$ lie inside the same sector, and $b_{1}$ is farther than $b_{2}$ from $u$. Since blue node $c_{1}$ covers $b_{1}$. Obviously, $c_{1}$ covers $b_{2}$ too. (This fact can be simply validated by geometry. Refer to Figure 6.) This is contradictory to that $b_{2}$ is covered only by $c_{2}$, since $b_{2}$ is covered by both $c_{1}$ and $c_{2}$. Therefore, the above assumption is false. This lemma is proved.

Algorithm 3 Reducing the number of blue nodes

Input: $G=(V, E)$; Sequence of MISs $B L A C K$; BFST $B T$; Sequence of blue node sets $B L U E$; Data aggregation tree $D T$.

Output: Reduced data aggregation tree based on $D T$.

1) Procedure ReduceBlueNodes ( $G, B L A C K, B T$, $B L U E, D T)$

2) $/ /$ Reduce the number of blue nodes layer by layer

3) FOR $i \leftarrow 0$ to $l-2 \mathbf{D O}$

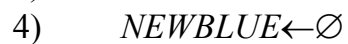

5) FOR each black nodes $u \in B L A C K_{i} \quad$ DO

6) Find $u$ 's blue children set $B C(u) \subseteq B L U E_{i+1}$

7) Find $u$ 's 2-hop black neighbors set $B N(u)$

8) Descending sort $B C(u)$ on the coverage density

9) WHILE $B N(u) \neq \varnothing$ DO

10) Get out a blue node $x$ from $B C(u)$

11) $\quad B C(u) \leftarrow B C(u)-\{x\}$

12) NEWBLUE $\leftarrow$ NEWBLUE $\cup\{x\}$

13) $\quad$ FOR each $w \in$ Coverage $(x)$ DO

14) Remove edge $\left(w, p_{D T}(w)\right)$ from

15) $E_{D T} \quad$ Add edge $(x, w)$ into $E_{D T}$

16) ENDFOR

17) $B N(u) \leftarrow B N(u)-$ Coverage $(x)$

18) ENDWHILE

19) ENDFOR

20) Color nodes in $B L U E_{i+1}-N E W B L U E$ from blue to white;

\section{ENDFOR}

RETURN $D T$

According to Lemma 3.1, we design Algorithm 3 to reduce the number of blue nodes. The idea of Algorithm 3 is based on a greedy strategy. For each black node $u \in$ $B L A C K_{i}$, we find $u$ 's blue children set $B C(u) \subseteq B L U E_{i+1}$ and $u$ 's 2-hop black neighbors set $B N(u)$, then we sort $B C(u)$ in a decreasing order on the coverage density. In the first repetition, we keep the blue node $x$ which has the largest coverage density, and remove $x$ from $B C(u)$. For each $w \in$ Coverage $(x)$, we remove edge $(w, p(w))$ from the data aggregation tree and add edge $(x, w)$ into the data aggregation tree. The covered black node set Coverage $(x)$ by blue node $x$ is removed from $B N(u)$. This process is terminated until $B N(u)$ is an empty set. Algorithm 3 is executed layer by layer. Finally, the rest blue nodes are converted from blue to white.

The pseudo code is presented in Algorithm 3. For convenience, the output of Algorithm 3 is called Reduced Data Aggregation Tree. Figure 7 shows an example of reducing the number of blue nodes based on the result in Figure 5.

To construct a data aggregation tree, we firstly execute Algorithm 2 to find a sequence of blue node sets $\left\{B L U E_{1}\right.$, $\left.B L U E_{2}, \ldots, B L U E_{l-1}\right\}$ that connect black nodes layer by layer and construct a data aggregation tree DT; Second, Algorithm 3 is executed to reduce the number of blue nodes layer by layer, and finally we obtain a data aggregation tree. The pseudocode of the entire procedure of data aggregation tree construction is presented in Algorithm 4.

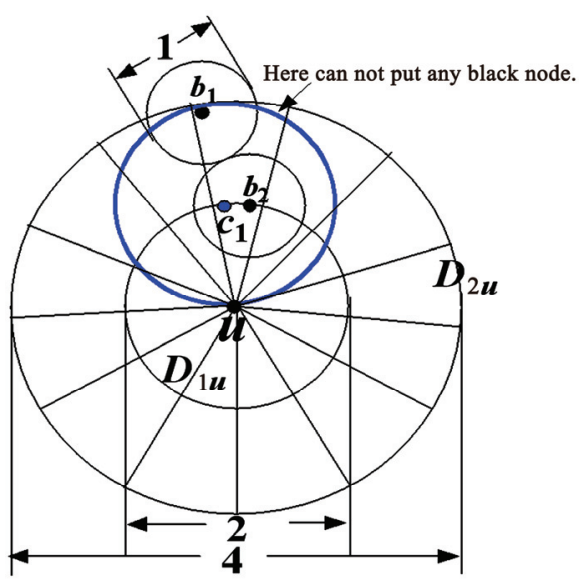

Figure 6. Proof of lemma 3.1.

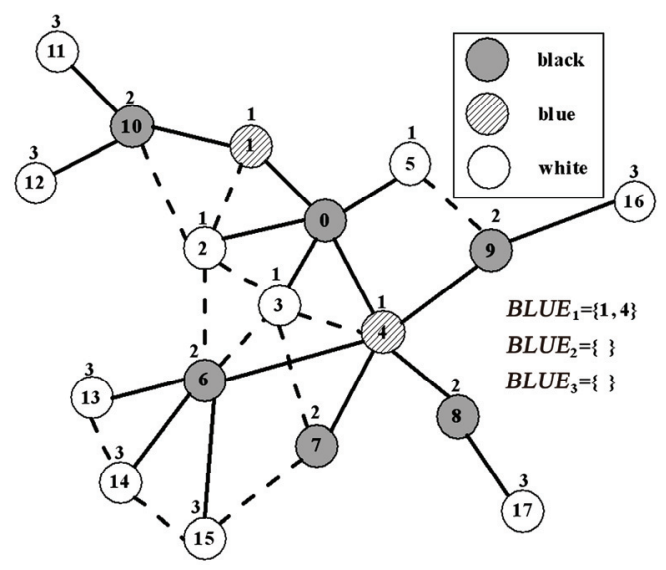

Figure 7. Reducing the number of blue nodes. 


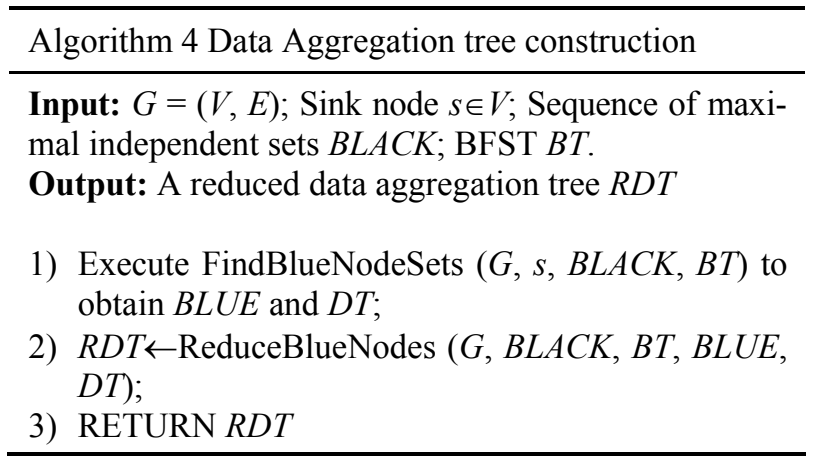

\subsection{Data Aggregation Scheduling}

In this section, we generate a data aggregation schedule based on the data aggregation tree. The process of generating a data aggregation schedule is simple and it breaks the transmission rule of white nodes sending to black nodes, black nodes sending to blue nodes and blue nodes sending to black nodes. The process of data aggregation scheduling is the process of cutting leaves.

The process of data aggregation scheduling takes in an input of a network topology $G=(V, E)$ and a corresponding data aggregation tree $D T$. First, we pick a node $u$ from the leaves of $D T$ and generate a transmission schedule $u \rightarrow p_{D T}(u)$, where $p_{D T}(u)$ is $u$ 's parent in $D T$, then we check whether $u \rightarrow p_{D T}(u)$ conflicts with any transmission schedule in the current non-conflicting transmission schedule set $S_{i}$ (its initial value is null, $i=1,2, \ldots$ ). If it does not conflict with any transmission schedule in $S_{i}$, then we add $u \rightarrow p_{D T}(u)$ to $S_{i}$. Otherwise, we check the next transmission schedule using a leaf of $D T$ as the sender. Finding a maximal non-conflicting transmission schedule set based on the leaves of $D T$ is similar with 0-1 knapsack problem and we present an approximate algorithm in Algorithm 5 due to it is NP-hardness. We do not prove the problem of finding a maximal non-conflicting transmission schedule set to be NP-hard due to page limitation. When we obtain a maximal non-conflicting transmission schedule set from the leaves of $D T$, we cut these leaves and edges associated with these leaves and their parents. This process is repeated until there is only one sink node $s$ in $D T$.

\begin{tabular}{|c|c|}
\hline \multicolumn{2}{|r|}{ Algorithm 5 Data Aggregation Scheduling } \\
\hline \multicolumn{2}{|r|}{$\begin{array}{l}\text { Input: } G=(V, E) ; \text { Sink } s \in V ; \text { Data aggregation tree } D T \\
\text { Output: Data aggregation schedule } S\end{array}$} \\
\hline 1) & $i \leftarrow 1 ; \quad S \leftarrow\{\varnothing\}$ \\
\hline 2) & WHILE there exists an edge in $D T$ \\
\hline 3) & $S_{i} \leftarrow \varnothing$ \\
\hline 4) & Leaves $\leftarrow\{u \mid u$ a is leaf of $D T\}$ \\
\hline 5) & WHILE Leaves $\neq \varnothing \quad$ DO \\
\hline & FOR each leaf $u$ in Leaves DO \\
\hline & FOR each $x \rightarrow p_{D T}(x) \in S_{i}$ DO \\
\hline
\end{tabular}

\begin{tabular}{lc}
\hline 8$)$ & IF $S_{i}=\varnothing$ or $u \notin$ Neighbor $(x)$ and \\
& $p_{D T}(x) \notin$ Neighbor $(u)$ \\
$9)$ & $S_{i} \quad \leftarrow S_{i} \cup\left\{u \rightarrow p_{D T}(u)\right\}$ \\
$10)$ & ENDIF \\
$11)$ & ENDFOR \\
$12)$ & ENDFOR \\
$13)$ & ENDWHILE \\
$14)$ & FOR each schedule $u \rightarrow p_{D T}(u)$ in $S_{i} \quad$ DO \\
$15)$ & Cut edge $\left(u, p_{D T}(u)\right)$ from $D T$ \\
$16)$ & ENDFOR \\
$17)$ & $S \quad S \cup S_{i} ; \quad i \leftarrow i+1 ;$ \\
$18)$ & ENDWHILE \\
\hline
\end{tabular}

The pseudo code of data aggregation scheduling is presented in Algorithm 5. Note that in Algorithm 5, we consider two types of collisions discussed in Figure 1, whereas the transmission rule of FIRSTFIT in [1] only considers type (a) in Figure 1.

As an example, given the reduced data aggregation tree in Figure 7, the final data aggregation schedule is given as follows: $S_{1}=\{11 \rightarrow 10 ; 13 \rightarrow 6 ; 7 \rightarrow 4 ; 17 \rightarrow 8 ; 5$ $\rightarrow 0 ;\} S_{2}=\{12 \rightarrow 10 ; 14 \rightarrow 6 ; 8 \rightarrow 4 ; 16 \rightarrow 9 ;\} S_{3}=$ $\{10 \rightarrow 1 ; 15 \rightarrow 6 ; 9 \rightarrow 4 ;\} S_{4}=\{1 \rightarrow 0 ; 6 \rightarrow 4 ;\} S_{5}=\{2$ $\rightarrow 0 ;\} S_{6}=\{3 \rightarrow 0 ;\} S_{7}=\{4 \rightarrow 0 ;\}$.

Let $L_{5-R D T}$ denote the data aggregation latency of Algorithm 5 . Then for this example we have $L_{5-R D T}=7$.

Lemma 3.2 For a given data aggregation tree $D T$, the data aggregation latency of Algorithm 5 is no more than that of Algorithm 4 in [1].

Proof: Algorithm 4 in [1] has the aptotic transmission rule of white nodes sending to black nodes, black nodes sending to blue nodes and blue nodes sending to black nodes. Nevertheless, our Algorithm 5 does not follow this rule. Algorithm 5 picks nodes from the leaves of the data aggregation tree $D T$ and generates transmission schedules. Furthermore, some black nodes are possibly leaves. For example, in Figure 7, node 7 is a black leaf.

Without loss of generality, for all the leaves of $D T$, there are $m$ white nodes and a black node $b$. According to the transmission rule of FIRSTFIT in [2], the $m$ white nodes and the black node $b$ are definitely in different sender sets $\operatorname{Sender}\left(S_{i}\right), \ldots, \operatorname{Sender}\left(S_{j-1}\right), \operatorname{Sender}\left(S_{j}\right)(i<j)$ such that $m$ white nodes are in $\operatorname{Sender}\left(S_{i}\right), \ldots, \operatorname{Sender}\left(S_{j-1}\right)$ and $b$ is in $\operatorname{Sender}\left(S_{j}\right)$, where $S_{i}, \ldots, S_{j-1}, S_{j}$ are transmission schedule sets. Moreover, according to the transmission rule of Algorithm 5, $m$ white nodes are in Sender $\left(S_{i}\right), \ldots, \operatorname{Sender}\left(S_{j-1}\right)$ and either $b$ is in $\operatorname{Sender}\left(S_{j}\right)$ if $b$ $\rightarrow p_{D T}(u)$ conflicts with transmission schedules in $S_{i}, \ldots$, $S_{j-1}$ or $b$ is in $\operatorname{Sender}\left(S_{k}\right)(i \leq k \leq j-1)$ if $b \rightarrow p_{D T}(u)$ does not conflict with transmission schedules in $S_{i}, \ldots, S_{j}$ ${ }_{-1}$. Thus, the data aggregation latency of Algorithm 5 is no more than that of Algorithm 4 in [1]. After cutting leaves from the current data aggregation tree, similar proof can be applied on the residual data aggregation tree.

For example, given the reduced data aggregation tree 
in Figure 7, the final data aggregation schedule based on FIRSTFIT in [1] is given as follows:

\section{WHITE to BLACK}

$S_{1}=\{5 \rightarrow 0 ; 11 \rightarrow 10 ; 13 \rightarrow 6 ; 17 \rightarrow 8 ;\}$

$S_{2}=\{16 \rightarrow 9 ; 14 \rightarrow 6 ; 12 \rightarrow 10 ; 3 \rightarrow 0 ;\}$

$S_{3}=\{15 \rightarrow 6 ;\}$

$S_{4}=\{2 \rightarrow 0 ;\}$;

BLACK to BLUE

$S_{5}=\{10 \rightarrow 1 ; 9 \rightarrow 4 ;\}$

$S_{6}=\{6 \rightarrow 4 ;\}$

$S_{7}=\{7 \rightarrow 4 ;\}$

$S_{8}=\{8 \rightarrow 4 ;\}$;

BLUE to BLACK

$S_{9}=\{1 \rightarrow 0 ;\}$

$S_{10}=\{4 \rightarrow 0 ;\}$.

Let $L_{4[2]-R D T}$ denote the data aggregation latency of Algorithm 4 in [1] taking in an input of a reduced data aggregation tree, then for this example we have $L_{4[2]-R D T}=$ $10>L_{5-R D T}=7$.

\subsection{Performance Analysis}

In this section we show that Algorithm 5 has a latency bound of $16 R+\Delta-11$. Suppose that $D T$ is a data aggregation tree generated by Algorithm 2 and $R D T$ is a reduced data aggregation tree generated by Algorithm 3 taking in an input of $D T$. Let $L_{4[2]-D T}$ denote the data aggregation latency of Algorithm 4 in [1] taking in an input of $D T, L_{4[2]-D T}=23 R+\Delta-18$; If we could estimate $L_{4[2]-R D T}=16 R+\Delta-11$, according to Lemma 3.2, there is $L_{5-R D T} \leq L_{4[2]-R D T}$, then $L_{5-R D T} \leq 16 R+\Delta-11$, i.e. Algorithm 5 has a latency bound of $16 R+\Delta-11$.

Now, let's estimate $L_{4[2]-R D T}=16 R+\Delta-11$. The transmission rule of Algorithm 4 in [1] has three parts: 1) WHITE to BLACK; 2) BLACK to BLUE; 3) BLUE to BLACK.

1) WHITE to BLACK: It takes at most $\Delta-1$ time slots [2] to finish the transmission.

2) BLACK to BLUE: It takes at most 4 time slots to finish the transmission.

3) BLUE to BLACK: We need to estimate how many blue nodes are competing to transmit to the same black parent. According to Lemma 3.1, we know that for a fixed black node $u \in B L A C K_{i}(i=0,1, \ldots, l-2)$, there are at most 13 blue nodes which can cover $u$ 's 2-hop black neighbors in $B L A C K_{i+2}$. One of these 13 blue nodes must be node $u$ 's parent. Therefore, there are at most 12 blue nodes which are competing to transmit to the same black parent.

WHITE-to-BLACK needs $\Delta-1$ time slots. From layer $R$ to layer 2, BLACK-to-BLUE and BLUE-to-BLACK together need $(12+4) \cdot(R-2)$ time slots. Transmission from layer 2 to layer 1 needs $13+4$ time slots and transmission from layer 1 to the sink needs 5 time slots. Altogether,

$$
\begin{aligned}
L_{4[2]-R D T} & =(\Delta-1)+(12+4) \cdot(R-2)+13+4+5 \\
& =16 R+\Delta-11 .
\end{aligned}
$$

Therefore, $L_{5-R D T} \leq L_{4[2]-R D T}<L_{4[2]-D T}=23 R+\Delta-18$, i.e. Algorithm 5 has a latency bound of $16 R+\Delta-11$.

Lemma 3.3 If $R \geq \Delta-11$, our algorithm has approximation ratio 17 .

Proof: Given a graph $G=(V, E)$ and the sink node $s \in$ $V$, let $L_{\text {opt }}$ denote the minimum data aggregation latency. Since farthest node has to transmit data to the sink, therefore $L_{\text {opt }} \geq R . L_{5-R D T} / L_{\text {opt }} \leq(16 R+\Delta-11) / R \leq 16+$ $(\Delta-11) / R$, when $R \geq \Delta-11, L_{5-R D T} / L_{\text {opt }} \leq 17$.

Lemma 3.4 If $R$ is enough large, our algorithm has approximation ratio 16 from an asymptotic point of view.

Proof: According to the proof of lemma 3.3, when $R$ tends to infinite, $(\Delta-11) / R$ tends to zero, therefore our algorithm has approximation ratio 16 from an asymptotic point of view.

For example, given a data aggregation tree $D T$ without reducing the number of blue nodes in Figure 5, the final data aggregation schedule generated by Algorithm 4 in [1] is given as follows:

WHITE to BLACK $S_{1}=\{5 \rightarrow 0 ; 11 \rightarrow 10 ; 13 \rightarrow 6$; $17 \rightarrow 8 ;\} S_{2}=\{16 \rightarrow 9 ; 14 \rightarrow 6 ; 12 \rightarrow 10 ;\} S_{3}=\{15 \rightarrow$ $6 ;\}$; BLACK to BLUE $S_{4}=\{10 \rightarrow 1 ; 9 \rightarrow 4 ;\} S_{5}=\{6 \rightarrow$ $2 ;\} S_{6}=\{7 \rightarrow 3 ;\} S_{7}=\{8 \rightarrow 4 ;\}$ BLUE to BLACK $S_{8}=$ $\{1 \rightarrow 0 ;\} S_{9}=\{2 \rightarrow 0 ;\} S_{10}=\{3 \rightarrow 0 ;\} S_{11}=\{4 \rightarrow 0 ;\}$.

In this example, we have $L_{4[2]-D T}=11$. For the example of the topology shown in Figure 2, we have: $L_{5-R D T}=$ $7<L_{4[2]-R D T}=10<L_{4[2]-D T}=11$.

\section{Simulation Results}

In this section, we evaluate our work by conducting extensive simulations. We compare our algorithm with Algorithm 4 in [1]. In the following, we first explain the objective of the simulations, the generation of a network and performance metric we used. Then we present the simulation results. There are two main factors that affect the data aggregation latency, network radius $R$ and the maximum degree of network topology $\Delta$. The objective of the simulations is straightforward, that is to investigate the effects of $R$ and $\Delta$ on the data aggregation latency. The performance metric is the number of transmission schedule sets in data aggregation schedule $\left\{S_{1}, S_{2}, \ldots, S_{l}\right\}$, i.e. the value of $l$. The smaller $l$ means that the better the algorithm performance.

We also implemented a topology simulator that can randomly deploy sensors into a square region of size $X \times X$, where $X$ is the width of the square. The topology simulator takes in an input of a network radius $R$, a maximum degree of network topology $\Delta$, a transmission range of each sensor node $r . R$ is the length of the longest path in data aggregation tree $D T$ from the sink to the leaf nodes of $D T$, i.e. the largest number of hops from the sink to 
the leaf nodes of $D T$. The output of this simulator is a topology which deploys $N$ sensor nodes randomly into a square region of size $X \times X$, where $N=(\Delta+1) R^{2} /(2 \pi)$, and $X=R \cdot r /$ sqrt (2.0). The deduction of $N$ and $X$ is based on the assumption that the node density $\rho$ is a constant such that in each node's transmission range there are $\Delta$ neighbors. Therefore, $\rho=(\Delta+1) /\left(\pi \cdot r^{2}\right), N=X^{2} \rho=(\Delta+$ $1) R^{2} /(2 \pi)$. Simulations are conducted from two points of view:

1) The sink is always the node nearest to the left-up corner. We investigate the effects of network radius $R$ and the maximum degree $\Delta$ on the data aggregation latency using the above topology simulator.

First, $\Delta$ is fixed to 20, and the transmission range $r$ is set to $30 \mathrm{~m}$. $R$ varies from 7 to 37 with an increment of 5 . In Figure 8, for each $R$, we generated 30 random topologies and computed the average data aggregation latency for 30 topologies. The average data aggregation latency is proportional to $R$. The pattern of those curves matched our theoretical estimation. Our algorithm has smaller latency compared with Algorithm 4 in [1] (denoted as Huang's algorithm).

Second, $R$ is fixed to 8 , and the transmission range $r$ is set to $30 \mathrm{~m}$. $\Delta$ varies from 18 to 63 with an increment of 5. As shown in Figure 9, for each fixed $\Delta$, we generated 30 random topologies and computed the average data aggregation latency for 30 topologies. The average data aggregation latency is also proportional to $\Delta$. Our algorithm has smaller latency compared with Huang's algorithm. Since $R$ is fixed, the gap between the two curves almost does not change. This is true according to our theoretical analysis.

2) The sink is always the node with ID 0. Its position is random. We randomly deployed sensors into a fixed region of size $400 \mathrm{~m} \times 400 \mathrm{~m}$. We investigated the effects of the number of nodes $N$ and the transmission range $r$ on the data aggregation latency.

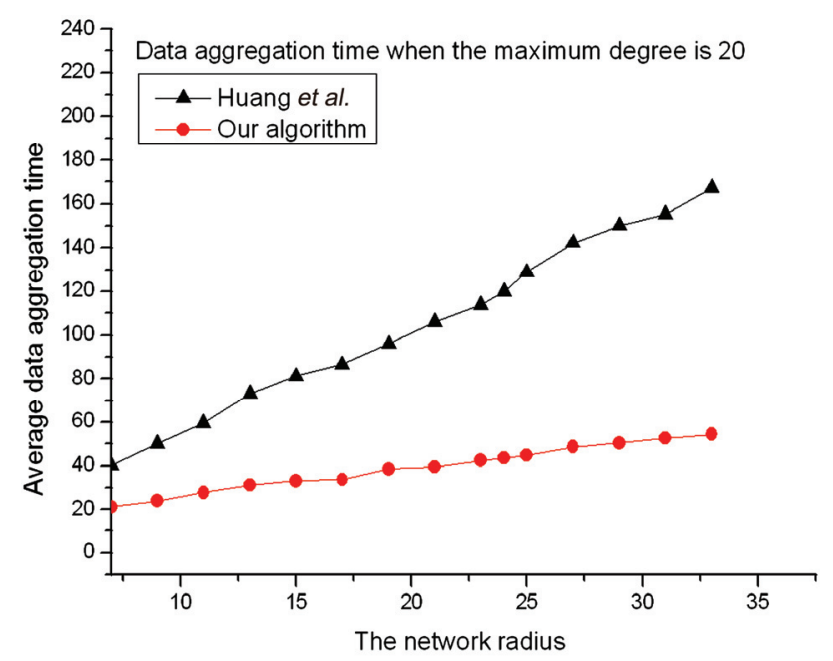

Figure 8. The effect of $R$.
First, the transmission range $r$ is set to $30 \mathrm{~m}$. N varies from 180 to 980 with an increment of 100. In Figure 10, for each $N$, we generated 30 random topologies and computed the average data aggregation latency for 30 topologies. When $N$ increases, $R$ and $\Delta$ both increase. $N$ is proportional to $(\Delta+1) R^{2}$. Our algorithm has smaller latency compared with Huang's algorithm.

Second, the number of sensor nodes $N$ is fixed to 200 . $r$ varies from 27 to 57 with an increment of 5. In Figure 11, for each $r$, we randomly deployed 200 sensors for 30 times and computed the average data aggregation latency for 30 topologies. In this simulation, the node density $\rho$ is a constant since a in fixed region there is a fixed number of sensor nodes. $R=\operatorname{sqrt}(2.0) \cdot X / r$, and $\Delta=\pi \cdot r^{2} \cdot \rho-1$. Obviously, when $r$ increases, $R$ decreases with $1 / r$ and $\Delta$ increases with $r^{2}$. Therefore, the two curves are nearly linear. Our algorithm has smaller latency compared with Huang's algorithm.

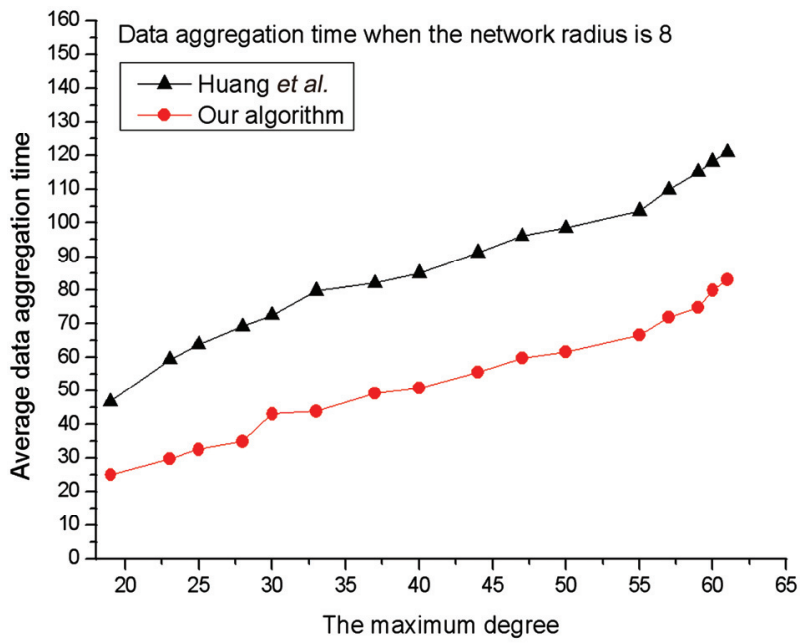

Figure 9. The effect of $\Delta$.

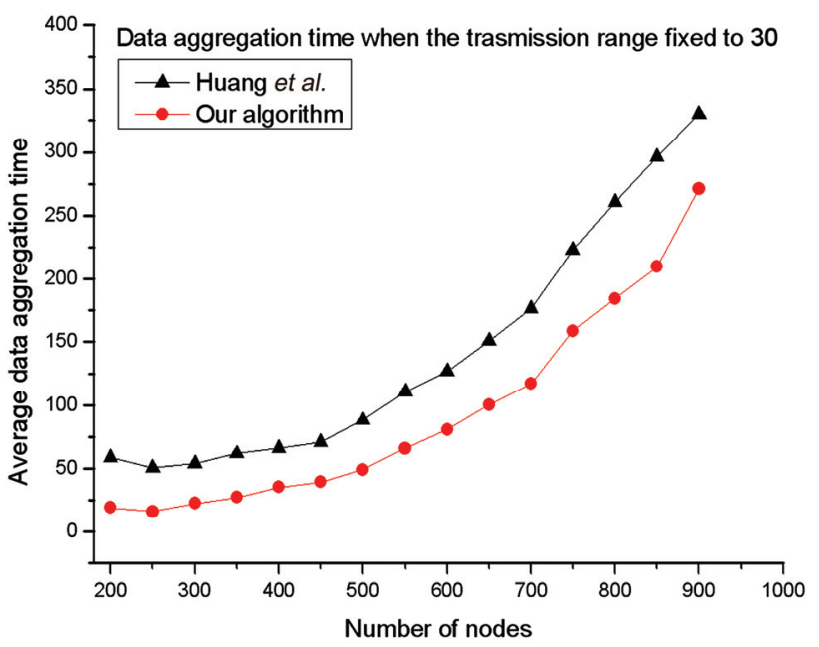

Figure 10. The effect of $N$. 


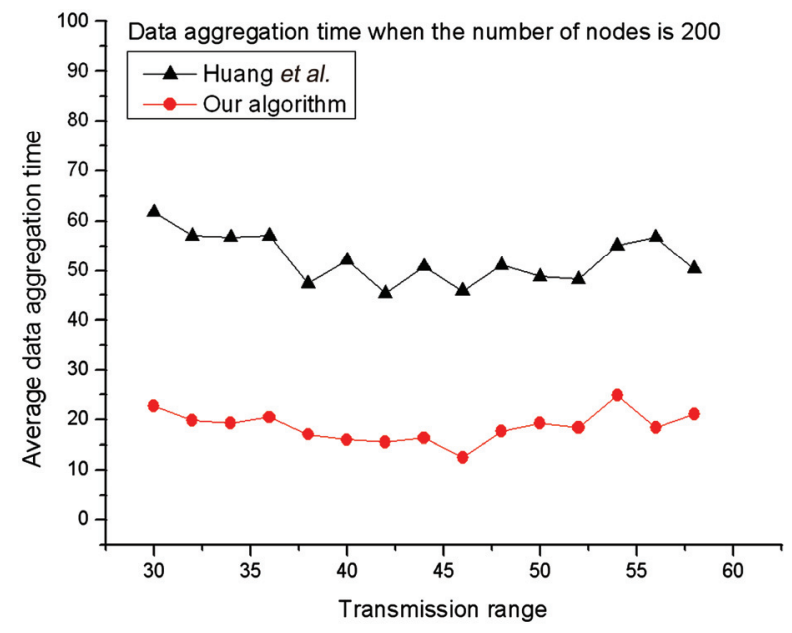

Figure 11. The effect of $r$.

\section{Conclusions}

The Existing works on data aggregation in WSNs usually use a single channel which results in a long latency due to high interference, especially in high-density networks. Therefore, data aggregation is a fundamental yet timeconsuming task in WSNs. In this paper, we investigate the minimum data aggregation latency problem. We used the techniques of reducing the number of blue nodes and finding maximal non-conflicting transmission schedule set based on leaves and designed an algorithm with a latency bound of $16 R+\Delta-11$, where $\Delta$ is the maximum degree and $R$ is the network radius. We prove that our algorithm has smaller latency than other algorithms. Simulation results show that our algorithm has much better performance in practice than previous works.

\section{References}

[1] S. C.-H. Huang, P.-J. Wan, C. T. Vu, Y. S. Li and F. Yao, "Nearly Constant Approximation for Data Aggregation Scheduling in Wireless Sensor Networks," Proceedings of 26th IEEE International Conference on Computer Communications, Anchorage, 6-12 May 2007, pp. 366372.

[2] X. J. Chen, X. D. Hu and J. M. Zhu, "Minimum Data Aggregation Time Problem in Wireless Sensor Networks," Proceedings of 1 st International Conference on Mobile Ad-Hoc and Sensor Networks, Lecture Notes in Computer Science, Wuhan, Vol. 3794, 13-15 December 2005, pp. 133-142.

[3] V. Annamalai, S. K. S. Gupta and L. Schwiebert, "On
Tree-Based Convergecasting in Wireless Sensor Networks," Proceedings of IEEE Wireless Communications and Networking, New Orleans, Vol. 3, 20 March 2003, pp. 1942-1947.

[4] S. Upadhyayula, V. Annamalai and S. K. S. Gupta, "A Low Latency and Energy-Efficient Algorithm for Convergecast in Wireless Sensor Networks," Proceedings of IEEE Global Telecommunications Conference, San Francisco, Vol. 6, 1-5 December 2003, pp. 3525-3530.

[5] H. J. Wu, Q. Luo and W. W. Xue, "Distributed CrossLayer Scheduling for in-Network Sensor Query Processing," Proceedings of 4th Annual IEEE International Conference on Pervasive Computing and Communications, Pisa, Vol. 10, 13-17 March 2006, pp. 180-189.

[6] O. Chipara, C. Y. Lu and J. Stankovic, "Dynamic Conflict-Free Query Scheduling for Wireless Sensor Networks," Proceedings of 14th IEEE International Conference on Network Protocols, Santa Barbara, 12-15 November 2006, pp. 321-331.

[7] Y. Yu, B. Krishnamachari and V. K. Prasanna, "Energy-Latency Trade-Offs for Data Gathering in Wireless Sensor Networks," Proceedings of 23rd Annual Joint Conference of the IEEE Computer and Communications Societies, Hong Kong, 7-11 March 2004, pp. 1-12.

[8] Q. F. Huang and Y. Zhang, "Radial Coordination for Convergecast in Wireless Sensor Networks," Proceedings of 29th Annual IEEE International Conference on Local Computer Networks, Tampa, 16-18 November 2004, pp. 542-549.

[9] H. W. Zhang, A. Arora, Y.-R. Choi and M. G. Gouda, "Reliable Bursty Convergecast in Wireless Sensor Networks," Proceedings of the 6th ACM International Symposium on Mobile Ad Hoc Networking and Computing, Urbana-Champaign, 25-28 May 2005, pp. 266-276.

[10] B. Krishnamachari, D. Estrin and S. B. Wicker, "The Impact of Data Aggregation in Wireless Sensor Networks," Proceedings of the 22nd International Conference on Distributed Computing Systems, Vienna, 2-5 July 2002, pp. 575-578.

[11] C. Intanagonwiwat, D. Estrin, R. Govindan and J. Heidemann, "Impact of Network Density on Data Aggregation in Wireless Sensor Networks," Proceedings of the 22nd International Conference on Distributed Computing Systems, Vienna, 2-5 July 2002, pp. 457-472.

[12] A. Kesselman and D. Kowalski, "Fast Distributed Algorithm for Convergecast in Ad Hoc Geometric Radio Networks," Proceedings of 2nd International Conference on Wireless on Demand Network Systems and Service, St. Moritz, 19-21 January 2005, pp. 1-6.

[13] R. A. Brualdi, "Introductory Combinatorics," 3rd Edition, Prentice Hall, Upper Saddle River, 1999, pp. 27-34. 